\title{
Implementation of a Force-Feedback Interface for Robotic Assisted Interventions with Real-time MRI Guidance.*
}

\author{
Nicholas C. von Sternberg, Atilla Kilicarslan, Nikhil V. Navkar, Zhigang Deng, \\ Karolos Grigoriadis, Nikolaos V. Tsekos
}

\begin{abstract}
Efficient and intuitive interfacing of the interventionalist to the information and tools available from image-guided robotic assisted surgeries is required to achieve the full benefit of these technologies. Ongoing research has been performed into the use of forbidden region guided fixtures (FRVF) for human-in-the-loop control of image-guided procedures via haptic force-feedback devices (FFD). Although commercially available FFD provide sufficient degrees-offreedom (DoF), collaborating clinicians, as well as the results of our previous work indicate that these systems are not completely intuitive for controlling fixed-point access interventional tool which have a remote center of motion. Within this context, we introduce a new FFD which is designed with the same DoF constraints as a fixed-point access interventional tool. The device is tested in a clinical simulation of a robot assisted trans-apical valve implantation under guidance from real-time magnetic resonance imaging. Preacquired real-time images are used in the clinical simulation to dynamically update the FRVF and therefore provide guiding forces to allow the operator to see the safe boundaries of operation via a visualization interface and physically feel them through the FFD. Inertial and gravity compensation and per DoF dynamic response of the physical prototype are validated and the frequency response of the system demonstrates it is adequate for tactile sensing. During clinical simulation the operator was successfully able to maneuver the tool within the safe path to the region of interest with the guidance of visual and force-feedback.
\end{abstract}

\section{INTRODUCTION}

Robotic assisted procedures are at the forefront of modern medicine due to their potential to increase treatment effectiveness and eventually reduce the overall cost of health care. Although the advent of minimally invasive procedures (MIP) has revolutionized patient treatment, it brought forward some new challenges for surgeons. The long, fixedaccess-point instruments used in MIP distance the hand from the area of procedure (AoP) which increases natural tremor, and reduces tactile sense, force-feedback, and visual perception [1, 2]. Robotic assistance overcomes these challenges not only through the capabilities of the endeffectors such as dexterity, accuracy, tremor removal, and

\footnotetext{
* This work was supported by the National Science Foundation under Grant CNS-0932272. All opinions, findings, conclusions or recommendations expressed in this work are those of the authors and do not necessarily reflect the views of our sponsors.

NCvS, NVN, ZD, and NVT are with the Department of Computer Science, University of Houston, Houston, TX, USA (E-mail: nvonsternberg@uh.edu, \{nvnavkar, zdeng,ntsekos\}@cs.uh.edu).

Atilla Kilicarslan and Karolos Grigoriadis are with Department of Mechanical Engineering, University of Houston, Houston, TX, USA.
}

force-scaling, but also through the robot-user interface itself, i.e. how the surgeon interacts with the robotic system $[3,4]$.

As more sophisticated methods of imaging are introduced for sensing the AoP, effective and intuitive immersion of the operator to a web of information becomes important to reduce work-load and complexity of operation. Within this context, the pursuit of enhanced human-in-the-loop guidance in manual or robot-assisted MIP, has led to the introduction of the concept of forbidden-region virtual fixtures (FRVF) and forbidden region guided fixtures $[5,6]$. The benefits of FRVF have been demonstrated for various applications, including orthopedic procedures [7], needle insertion [8], medical diagnosis [9, 10], and image analysis [11]. Pioneering works have illustrated the power of combining imaging with FRVF $[12,13]$. Real-time imaging, such as real-time magnetic resonance imaging (rtMRI), provides the opportunity for dynamic FRVF [14] from which forces are exerted via a force-feedback device (FFD) to guide an operator in maneuvering an interventional tool.

Several commercial haptic devices are available including the six degree-of-freedom (DoF) PHANTOM OMNI (Geomagic-Sensable Group, USA) and the 5 DoF Haptic Wand (Quansar, USA). Although these systems provide the necessary DoF and range-of-motion for control of MIP, their kinematics, ergonomics and eventually intuitiveness are among the factors of their limited use in MIP. One noticeable difference between the use of these haptic devices and the tools used in interventional procedures is hand grip. Stylus and wand style haptic devices require a grip which requires different motion and muscles of the wrist [15]. A study by Lai et al. [16] demonstrated that an operator exhibits lower error and lower workload in a system where the master device has the same DoF and motion constraints as the slave device. Therefore it is beneficial to optimize motion constraints in addition to range-of-motion. These considerations, as well as feedback from our previous work [14] and direct input from collaborating clinical interventionists in radiology and cardiology have lead us to develop a new FFD to function as a master manipulator to control actuated interventional tools.

Unlike existing general purpose FFD, this master manipulator is designed specifically to provide an intuitive, ergonomic, and familiar experience to the operator. The 5 DoF system is configured to better model an interventional tool in feel and effective motion by constraining the first four DoF to restrict motion in the same way as an interventional tool through a fixed entry point (such as a trocar). The fifth DoF controls angulation of the distal tip which is used in our 
clinical simulation of robot assisted transapical valve implantation (TAVI). Forces are generated based upon dynamic FRVF generated from rtMRI feeds via a multithreaded data processing pipeline [14]. The methods of this design including: kinematic structure, system architecture, ergonomics, image-processing, visual force-feedback, and inertial compensation are discussed within. Results are reported in the form of characterization, dynamic performance, and a clinical simulation of a physical prototype.

\section{METHODS}

\section{A. Overview}

The FFD described herein is the master device for our system for Multimodal Image-guided RObot-assisted Surgeries (MIROS). The clinical paradigm for MIROS is MRI-guided robotic TAVI on the beating heart, which is demonstrated by Walther et al. [17] as a single-port access procedure. Fig. 1(a) illustrates a model of a generalized slave manipulator which consists of a straight tubular link with a bending section near its distal tip. This manipulator was derived in prior TAVI simulation studies [18] to be suitable for intracardiac procedures via a transapical access. Although the master device is designed for the TAVI slave manipulator, the principles used for this design are appropriate for other fixed-point access procedures with remote center of motion.

\section{B. Kinematic Structure}

The FFD has five DoF (Fig. 1b) which directly correspond to the five DoF of the slave device (Fig. 1a). The first four DoF, yaw $\left(\theta_{1}\right)$, pitch $\left(\theta_{2}\right)$, translation $\left(\mathrm{d}_{3}\right)$, and roll $\left(\theta_{4}\right)$, have intersecting axes which define a remote center of motion that directly corresponds to the center of the access port in the patient's body. The fifth DoF is added to the handle of the device to control the angulation of the distal tip angulation $\left(\theta_{5}\right)$ of the slave manipulator using a rotating paddle. The paddle is curved so that it can be comfortably actuated using abduction and adduction of the thumb. All 5 DoF are actuated by one hand, as requested by our clinicians, so that the operator has one hand free to perform other tasks.

\section{Ergonomics}

To provide a familiar and comfortable feel to surgeons, the handle of this FFD was modeled to be similar to the handle of a laparoscopic device. However, to provide a good surface area for grip the handle is designed to be larger than

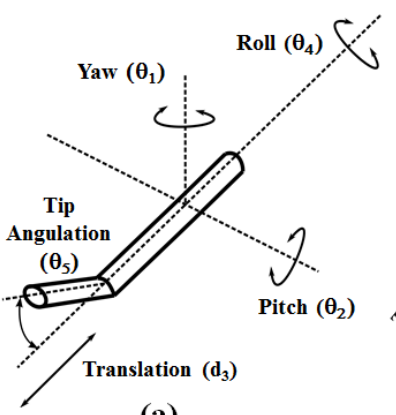

(a)

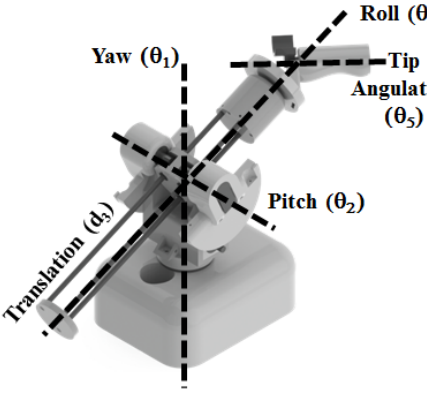

(b)
Fig. 1: (a) The interventional tool used as the basis of the FFD design (b) CAD model of the FFD with corresponding DoF illustrated.

a standard laparoscopic tool. The handle is contoured to fit the shape of the hand with a diameter ranging between 35 $\mathrm{mm}$ and $45 \mathrm{~mm}$ and the end is pommeled to prevent a loss of grip. These features were added as a result of the ergonomic guidelines set forth by Patkin in [15] and should allow a familiar and intuitive feeling for the interventionalist, but with a more comfortable grip with reduced fatigue compared to a standard laparoscopic handle.

\section{System Architecture}

The system architecture of the guidance interface (Fig. 2) is composed of several independent modules including the operator, force-feedback device, force-feedback controller (implemented on a dSPACE system), and a dedicated host PC. The dedicated host PC has the following sub-modules which are executed as independent threads: collision detection and response, image processing, visualization, and kinematics. Since the system is not fully automated, the operator is an essential component of the kinematic control loop which is indicated by the solid lines of Fig. 2. In this human-in-the-loop control, the operator uses the FFD to command desired motion while he or she receives feedback as force and visual cues.

\section{E. MRI Processing and Forbidden Region Calculation}

The MR images are processed on-the-fly using an interleaved multi-slice imaging protocol presented in [14] to generate a virtual access corridor which represents tissue boundaries throughout the AoP. The dynamically updated access corridor is then used as a FRVF to guide the operator to stay within an appropriate safe-distance of the corridor boundaries. The corridor is integrated with real-time telemanipulated robot control by implementing a multithreaded processing core to generate feedback force.

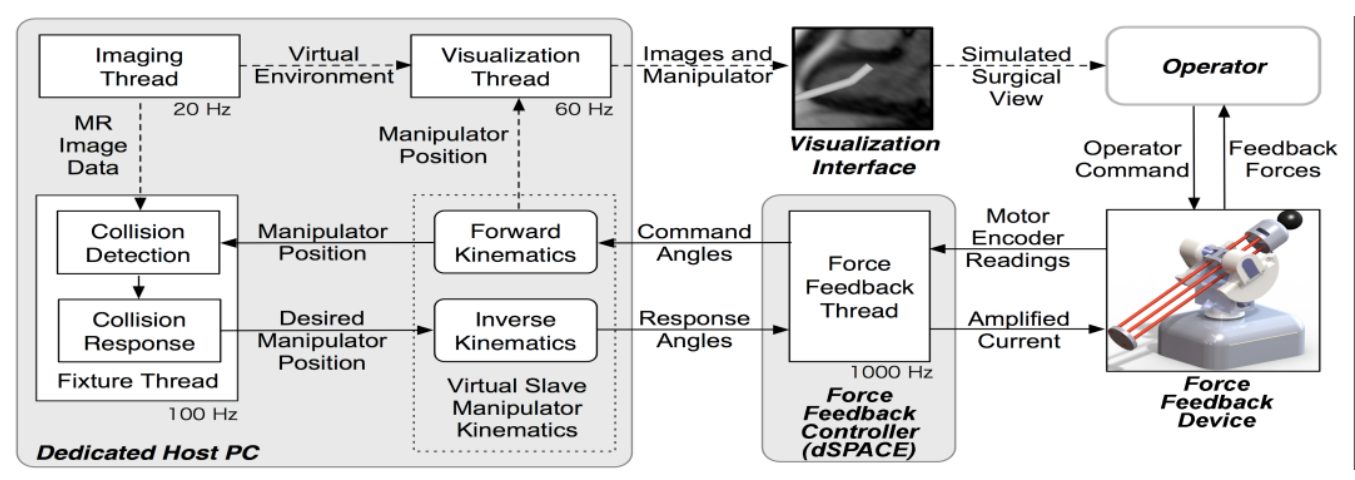

Fig. 2: System architecture. The solid lines form a loop which includes the operator creating human-in-the-loop control 


\section{F. Visual and force-feedback}

To provide visual feedback, the current position and orientation of the interventional tool is displayed over realtime MRI images of the beating heart (Fig. 3). Encoder signals from the FFD are used to update the visual feedback, as well as check for collision with the forbidden-region. In the case that the operator moves the tool beyond the perimeter of the safe access corridor, a collision is detected and handled using the process described in [14]. Collision with the FRVF results in forces exerted by the FFD to encourage the operator to adjust the tool position to a safer location, but zero force is exerted within the safe-access corridor. The reaction force $F_{c}$ is calculated as follows for each DoF:

$$
F_{c}=K_{d} R_{e}+B_{d} \dot{R}_{e}
$$

where the parameters $K_{d}$ and $B_{d}$ are the virtual stiffness and damping coefficients respectively and $R_{e}$ is the penetration distance on the relevant axis. Calculated force values are then converted to torque values and eventually drive currents.

\section{G. Gravity and Inertia Compensation}

As a result of the serial kinematic chain used in this device, the yaw $\left(\theta_{1}\right)$, pitch $\left(\theta_{2}\right)$, and roll $\left(\theta_{4}\right)$ DoF in this system have varying inertia which depends on the orientation and load distribution of subsequent joints with respect to the gravitational field. This inertia manifests itself in the form of undesired and non-uniform resistance to the user while manipulating the FFD. The force generation module incorporates a compensation routine which cancels these inertial effects depending on the system pose. Using encoder information, the resistive torques can be estimated as a result of the distributed masses of subsequent links along the relevant axis for each DoF and the respective cancellation currents can be calculated.

\section{RESULTS}

\section{A. Workspace}

Fig. 4 illustrates the reachable workspace in $\mathrm{mm}$ for the introduced FFD device. This corresponds to variations of the parameter vector $\left(\theta_{1}, \theta_{2}, \mathrm{~d}_{3}, \theta_{4}, \theta_{5}\right)$ by an iterative approach for discrete joint angle variations bounded by operational joint limits and calculating the end-effector position using forward kinematics.

\section{B. Physical Prototype}

The current physical prototype (Fig. 5), which is $53 \mathrm{~cm}$ long and $27 \mathrm{~cm}$ tall, was constructed from a combination of off-the-shelf and 3d-printed ABS parts. Physical prototyping went through several iterations to select specific design

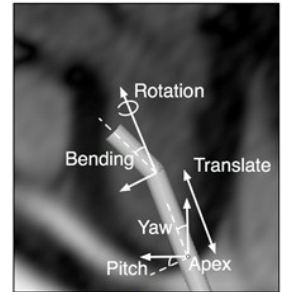

(a)

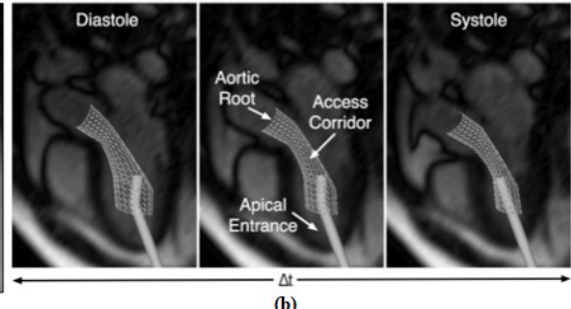

(b)
Fig. 3: a) Virtual tool rendering. b) Operation within the safe access corridor.

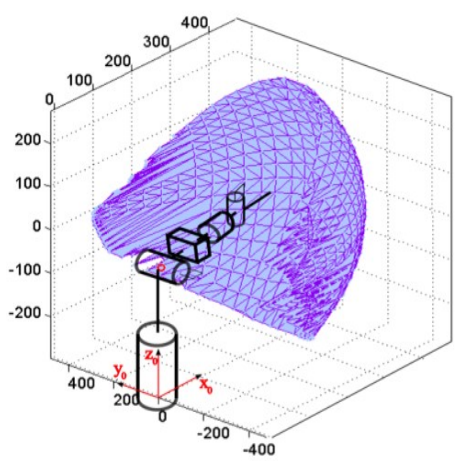

Fig. 4 - Maximum workspace $(\mathrm{mm})$ of possible end effector positions. details and physical components to balance three aspects: (i) a rigid and stiff structure, (ii) lightweight as possible for low inertia and gravity, and (iii) minimal friction. To achieve realistic force perception of the dynamic guidance virtual fixtures, direct cable drives and capstan mechanisms were selected to minimize inertia, friction, gravitational forces, and backlash. Such mechanisms are well documented in literature [19] and are also in use in commercial products such as the PHANTOM-Omni.

In total, seven brushed DC motors were used for this device since two motors were used to actuate each the pitch and translation DoF. Because the yaw DoF directly supports the majority of the mass of the FFD, a MCG-3040 brushless DC motor (Motion Control Group) was selected featuring $2598 \mathrm{Nmm}$ stall-torque and a $5000 \mathrm{cpr}$ optical encoder (Renco). The remaining six motors used for the other four DoFs are RE-max29 brushless DC motors (Maxon Motors) featuring $262 \mathrm{Nmm}$ stall-torque motors and $512 \mathrm{cpr}$ encoders. Motion, force output, and position resolution characteristics are listed in Table 1.

\section{Frequency Response}

Frequency response is a crucial performance measure of the dynamic performance e.g. the inertial, stiffness, and friction properties of the FFD [20]. Since this device is a multi-DoF system, its frequency response depends on different configurations of the system. For instance, the dynamics of the yaw angle $\left(\theta_{1}\right)$ would change slightly as a function of the translation distance $\left(d_{3}\right)$ since the center of mass would be shifted. Several conditions were tested by constraining certain DoF to a specific pose. Response to harmonic motion was recorded for each condition over an increasing frequency up to $14 \mathrm{~Hz}$ for several different input

TABLE I

OUTPUT FORCE AND POSITION RESOLUTION

\begin{tabular}{|c|c|c|c|c|}
\hline DoF & $\begin{array}{c}\text { Maximum } \\
\text { Continuous } \\
\text { Force } \\
\text { Output [N] }\end{array}$ & $\begin{array}{c}\text { Change of } \\
\text { Motion } \\
\text { [Deg] }\end{array}$ & $\begin{array}{c}\text { Minimum } \\
\text { Position } \\
\text { Resolution } \\
\text { [Deg] }\end{array}$ & $\begin{array}{c}\text { End- } \\
\text { Effector } \\
\text { Position } \\
\text { Resolution } \\
\text { [m m] }\end{array}$ \\
\hline $\boldsymbol{\theta}_{\boldsymbol{1}}$ & \pm 6.21 & \pm 80 & 0.072 & 0.260 \\
\hline $\boldsymbol{\theta}_{\boldsymbol{2}}$ & \pm 11.28 & \pm 42 & 0.047 & 0.170 \\
\hline $\boldsymbol{d}_{\boldsymbol{3}}$ & \pm 53.18 & $\pm 175.5^{\mathrm{b}}$ & 0.047 & 0.057 \\
\hline $\boldsymbol{\theta}_{\boldsymbol{4}}$ & \pm 10.48 & \pm 360 & 0.700 & $0.360^{\mathrm{a}}$ \\
\hline $\boldsymbol{\theta}_{\boldsymbol{5}}$ & \pm 13.10 & \pm 110 & 0.047 & 0.024 \\
\hline
\end{tabular}




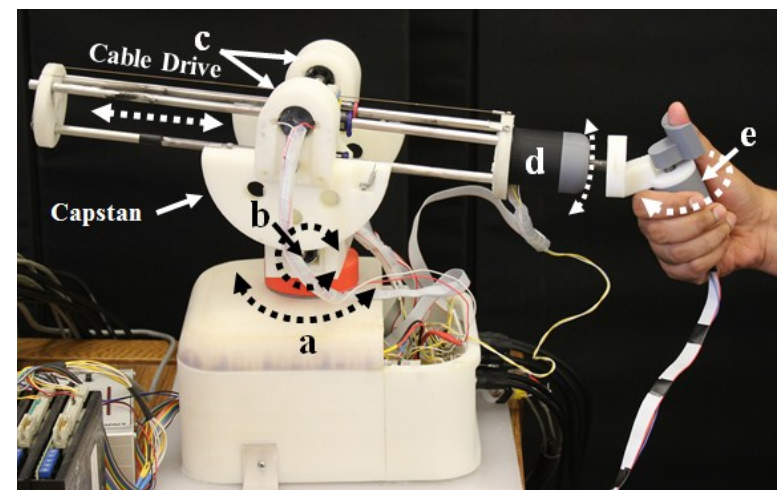

Fig. 5 - Working prototype with location of the yaw (a), pitch (b), translation (c), roll (d), and angulation (e) motors marked accordingly signal amplitudes. Because this system has no intrinsic spring element, a PD controller was required to prevent drift as suggested by Kim et al. [21].

Experimental conditions, pose constraints, and input signal amplitudes for each DoF are summarized in Table 2 and Fig. 6 shows the frequency responses of the FFD for the given parameters and conditions. As expected, the observed cut-off frequency decreases for the unbalanced conditions when $d_{3}=70 \mathrm{~mm}$ for yaw (Fig. 6a) and pitch (Fig. 6b). As in [21], the resulting frequency response is presented as magnitude scaled by the motors' continuous torque and the vibration threshold of the human hand [22] is presented for reference. Although the primary feature of this device is to generate an assisting force-feedback to the operator, not tactile sensing, comparisons with the vibration detection threshold of the human hand can be used for the evaluation of the device's performance. As shown in Fig. 6, the vibration detection threshold was shown to be greater than that of the human hand for all configurations tested.

\section{Gravity and Inertia Compensation}

Gravity and inertia compensation was implemented on the yaw, pitch, and roll, axes. Fig. 6 shows results from inertia compensation on the yaw axis. In this experiment, the FFD was used to control the motion of a virtual slave-manipulator
T ABLE II

FREQUENCY RESPONSE TEST CONDITIONS

\begin{tabular}{|l|l|l|l|}
\hline \multirow{2}{*}{$\begin{array}{l}\text { Degree of } \\
\text { Freedom }\end{array}$} & $\begin{array}{l}\text { Configuration Parameters } \\
\text { Reference } \\
\text { Signal }\end{array}$ & $\begin{array}{l}\text { Reference } \\
\text { Amplitudes }\end{array}$ & $\begin{array}{l}\text { Pose } \\
\text { Constrain ts }\end{array}$ \\
\hline Yaw $\left(\boldsymbol{\theta}_{1}\right)$ & $\theta_{1}=a \sin (\omega t)$ & $a=[2,8]$ & $\begin{array}{l}\theta_{2}=0 \\
d_{3}=[0,70]\end{array}$ \\
\hline Pitch $\left(\boldsymbol{\theta}_{2}\right)$ & $\theta_{2}=a \sin (\omega t)$ & $a=[2,8]$ & $\begin{array}{l}\theta_{2}=-20 \\
d_{3}=[0,70]\end{array}$ \\
\hline $\begin{array}{l}\text { Translation } \\
\left(\boldsymbol{d}_{3}\right)\end{array}$ & $d_{3}=a \sin (\omega t)$ & $a=[20,30,40]$ & $\theta_{2}=0$ \\
\hline Roll $\left(\boldsymbol{\theta}_{4}\right)$ & $\theta_{3}=a \sin (\omega t)$ & $a=45$ & -- \\
\hline $\begin{array}{l}\text { Tip-Angle } \\
\left(\boldsymbol{\theta}_{5}\right)\end{array}$ & $\theta_{4}=a \sin (\omega t)$ & $a=45$ & -- \\
\hline
\end{tabular}

inside a static virtual wall (i.e., a unilateral constraint). Fig. 7 presents the recorded yaw angle and control current over a 15 second trial period. Without compensation, the control current should be zero until the virtual-slave end effector makes contact with the virtual wall as shown during the 7 to 11 second time period in Fig. 7. The compensation effect is observed before and after this period as the cycling current which is only present in the case which inertia compensation was active.

\section{E. Clinical Simulation}

The developed FFD was tested for performing MRIguided procedures in a dynamic AoP for the simulated clinical scenario for TAVI [17, 18, 23]. This particular clinical paradigm was selected as a representative application to assess how the FFD would be used in presence of a highly dynamic environment. To perform offline simulation, previously recorded rtMRI feeds of a beating heart were fed to the control module at a frequency of $50 \mathrm{~ms}$ to represent image collection and transfer time. The virtual slave robot (Fig. 3a) was implemented as a rigid graphical object with the same dimensions as a surgical tool that would be used in an actual TA-AVI procedure. With the use of the FFD, the distal portion of the virtual robot was maneuvered by the operator to be placed and centered in the aortic root for delivering a virtual prosthetic valve.

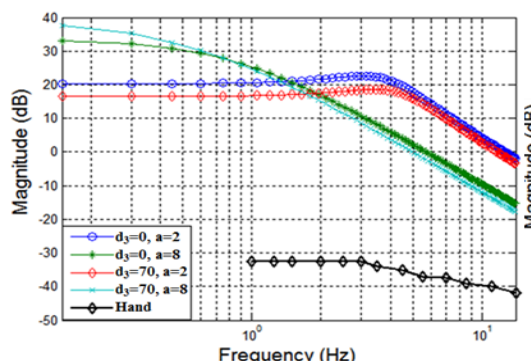

(a)

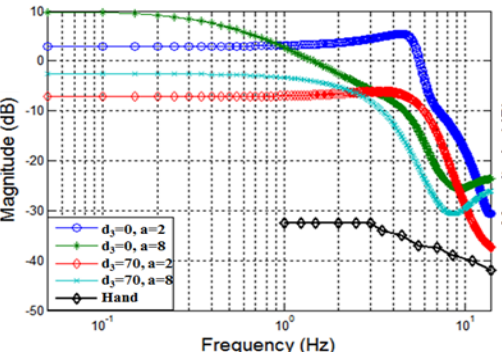

(b)

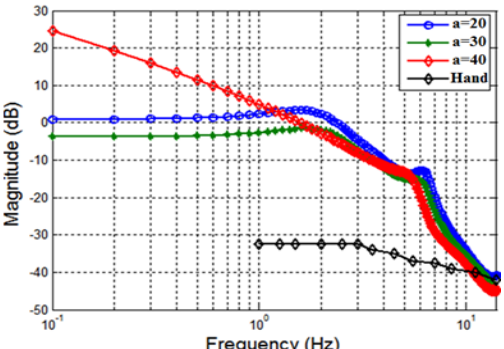

(c)

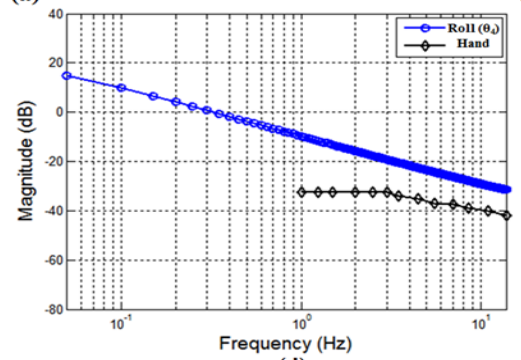

(d)

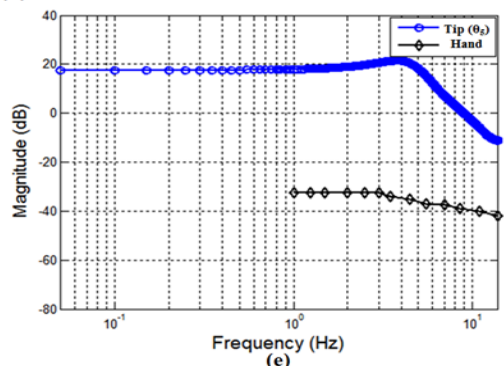

(e)

Fig. 6: Scaled frequency Response for (a) yaw (b) pitch (c) translation (d) roll (e) tip-angulation compared to vibration threshold of human hand 

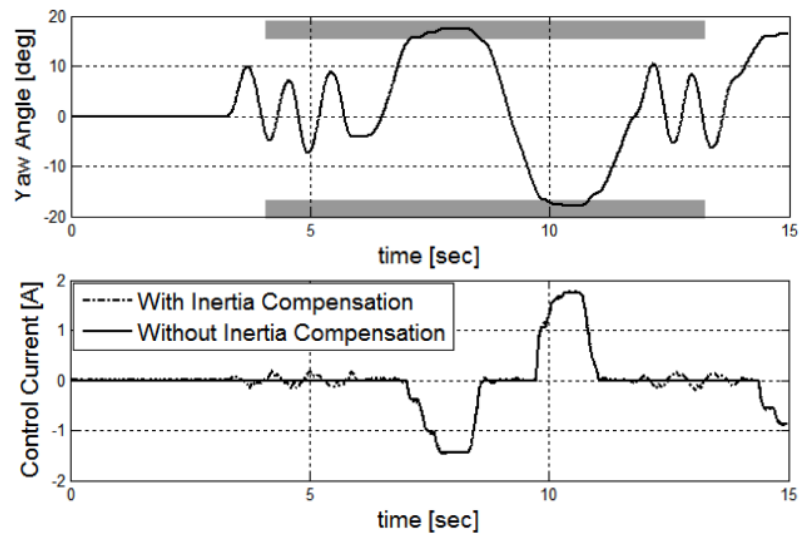

Fig. 7: Current foryaw $\left(\theta_{l}\right)$ with and without inertial compensation.

In these studies, the command and the response angles, exchanged between the simulation system (Host PC) and the FFD Controller (dSPACE), were continuously recorded during manipulation. Fig. 8 shows example results from such studies, where the red lines represent command angles (bending and rotational) from the FFD Controller to the simulation system and the blue lines show the response angles (bending and rotational) from the simulation system to the FFD Controller. A difference between the blue and red lines indicates that the operator attempted to command the slave-robot into the forbidden region, but was guided by the FFD. This is confirmed by the collision detected data at the bottom of Fig. 8. It is shown that the feedback forces generated by the FFD device assist the operator to stay inside the dynamic safe corridor, thus completing the human-in-the-loop-control.

\section{Discussion}

This work introduces a new force-feedback interface for use as the master-manipulator for rtMRI-based human-inthe-loop control of slave-manipulators. This system is the result of prior work focused on the development and integration of enabling methodologies in MRI-guided and robot-assisted intracardiac procedures $[14,18]$. Studies with collaborating clinicians clearly demonstrated that the human-machine interface could be substantially improved in terms of intuitiveness and ergonomics. Based on this feedback, the device was designed with kinematic structure and ergonomics so the operator manipulates the device in a similar manner with a fixed-point access tool. This provides a familiar and intuitive experience for the interventionalist. Although four DoF are required to maneuver an interventional tool through a fixed-point access point, this FFD was endowed with a fifth DoF to control the bendable

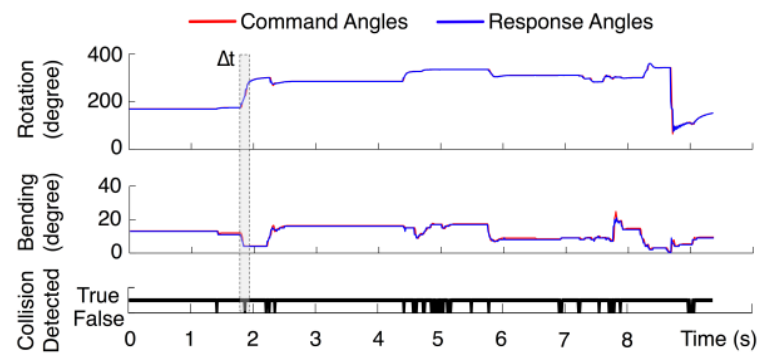

Fig. 8: Command and response angles for roll (Rotation) and tip angulation (Bending). "Collision Detected" indicates that the end effector was commanded to enter the forbidden region. distal portion of a slave manipulator. For use in other applications, this actuated thumb paddle could be exchanged with a trigger to resemble grasper and scissor type tools.

Feedback forces were calculated based upon the dynamic virtual fixtures (extracted from rtMRI) in order to guide the safe maneuvering of a slave manipulator. The exerted forces were therefore calculated relative to position error and the corresponding spring and damper force coefficients $\mathrm{K}_{\mathrm{d}}$ and $B_{d}$ were found heuristically. In the future, this system could be expanded to incorporate tool-to-tis sue interaction with the addition of force sensors.

The prototype described within this text was tested and deemed suitable by the collaborating cardiologists and surgeons who also participated in its original design. The results of the bench-top studies demonstrated a vibration threshold greater than that of the human hand. Continuous force output of each DoF was greater than that of the commercially available haptic devices surveyed except the yaw DoF. In light of these results, the yaw DoF can be improved with the use of a capstan pulley similar to the one used in the pitch DoF (which amplified torque 15 times).

Although preliminary results demonstrated the potential benefits of the FFD for guiding interventions using on-thefly rtMRI feeds, there were some associated limitations. First our studies used a virtual slave manipulator in place of an actual robotic slave. However, the kinematics of the virtual manipulator were modeled after the slave manipulator described in Fig 1(a). Second, the device was tested by a rather small sample size of clinicians $(n=3)$. Future studies will focus on recruiting a larger sample of interventional radiologists and surgeons to further systematically assess and fine-tune its functionality and ergonomics accordingly. Third, our computational core used pre-acquired feeds of rtMRI rather than being tested on-line with a MR scanner. These offline tests enabled bench testing and addressed logistics with the availability of clinicians and MR scanners. Since both the imaging and FFI threads behaved and tested under realistic timing conditions, this setup does not practically affect these particular studies.

While the motivation of this work was the use of the FFI to perform interventions with rtMRI guidance, it should be noted that the computational core of this system, as well as the FFD itself were designed in a modular fashion so that they could be used in, or exchanged with, other systems. Future work is planned for the integration of this master manipulator with an MRI compatible robotic manipulator which is under development.

\section{CONCLUSION}

This work presents a 5 DoF force-feedback interface as the master manipulator for controlling the yaw, pitch, translation, axial rotation, and distal-tip bending of a slave manipulator. This specific kinematic structure was pursued to provide intuitive operation by replicating the motion constraints of the actual device. Ergonomics were carefully considered to provide familiarity and reduce fatigue to the operator. This interface was tested on the paradigm of performing image-guided intracardiac procedures via transapical access utilizing rtMRI feeds to generate dynamic 
virtual fixtures which are used to exert forces to the operator. Visual and force-feedback were successfully used by the operator for guiding and maneuvering the slave manipulator to a target inside the beating heart.

\section{REFERENCES}

[1] A. Grimbergen, J. E. N. Jaspers, J. L. Herder, and C. H. G. Stassen, "Development of laparoscopic instruments," Minimally Invasive Therapy \& Allied Technologies, vol. 10, pp. 145-154, 2001.

[2] J. P. Ruurda, T. h. J. M. V. van Vroonhoven, and I. A. M. J. Broeders, "Robot-assisted surgical systems: a new era in laparoscopic surgery," Annals of the Royal College of Surgeons, vol. 84, pp. 223-226, 2002.

[3] E. J. Hanly and M. A. Talamini, "Robotic abdominal surgery," The American Journal of Surgery, vol. 188, pp. 19-26, 2004.

[4] P. Stadler, L. Dvoracek, P. Vitasek, and P. Matous, "Robotic vascular surgery, 150 cases," The International Journal of Medical Robotics and Computer Assisted Surgery, vol. 6, pp. 394-398, 2010.

[5] J. J. Abbott, P. Marayong, and A. M. Okamura, "Haptic Virtual Fixtures for Robot-Assisted Manipulation," in 12th International Symposium of Robotics Research (ISRR\}, 2005, pp. 49-64.

[6] A. M. Okamura, "Methods for Haptic Feedback in Teleoperated Robot-Assisted Surgery," Industrial Robot An International Journal, vol. 31, pp. 499-508, 2004.

[7] M. Agus, G. J. Brelstaff, A. Giachetti, E. Gobbetti, G. Zanetti, A. Zorcolo, B. Picasso, and S. S. Franceschini, "Physics-based burr haptic simulation: tuning and evaluation," presented at Haptic Interfaces for Virtual Environment and Teleoperator Systems, 2004. HAPTICS '04. Proceedings. 12th International Symposium on, 2004.

[8] C. Simone and A. M. Okamura, "Modeling of needle insertion forces for robot-assisted percutaneous therapy," presented at Robotics and Aut omation, 2002. Proceedings. ICRA '02. IEEE International Conference on, 2002.

[9] A. Kotranza and B. Lok, "Virtual Human + Tangible Interface $=$ Mixed Reality Human An Initial Exploration with a Virtual Breast Exam Patient," presented at Virtual Reality Conference, 2008. VR '08. IEEE, 2008.

[10] R. L. Williams, M. Srivastava, J. N. Howell, J. Robert R. Conatser, D. C. Eland, J. M. Burns, and A. G. Chila, "The virtual haptic back for palpatory training," in Proceedings of the 6th international conference on Multimodal interfaces. State College, PA, USA, 2004, pp. 191-197.

[11] S. Senger, "Integrating haptics into an immersive environment for the segmentation and visualization of volumetric data," presented at Eurohaptics Conference, 2005 and Symposium on Haptic Interfaces for Virtual Environment and Teleoperator Systems, 2005. World Haptics 2005. First Joint, 2005.

[12] M. Li, M. Ishii, and R. H. Taylor, "Spatial Motion Constraints Using Virtual Fixtures Generated by Anatomy," Robotics, IEEE Transactions on, vol. 23, pp. 4-19, 2007.

[13] R. Jing, R. V. Patel, K. A. McIsaac, G. Guiraudon, and T. M. Peters, "Dynamic 3-D Virtual Fixtures for Minimally Invasive Beating Heart Procedures," Medical Imaging, IEEE Transactions on, vol. 27, pp. 1061-1070, 2008.

[14] N. Navkar, Z. Deng, D. Shah, and N. Tsekos, "A Framework for Integrating real-time MRI with Robot Control: Application to Simulated Transapical Cardiac Interventions," Biomedical Engineering, IEEE Transactions on, vol. PP, pp. 1-1, 2012.

[15] M. Patkin, "Ergonomic aspects of surgical dexterity," The
Medical Journal of Australia, vol. 2, pp. 775-7, 1967.
[16] F. Lai and R. D. Howe, "Evaluating control modes for

[15] M. Patkin, "Ergonomic aspects of surgical dexterity," The
Medical Journal of Australia, vol. 2, pp. 775-7, 1967.
[16] F. Lai and R. D. Howe, "Evaluating control modes for constrained robotic surgery," presented at Robotics and Aut omation, 2000. Proceedings. ICRA '00. IEEE International Conference on, 2000.

[17] T. Walther, T. Dewey, M. A. Borger, J. Kempfert, A. Linke, R. Becht, V. Falk, G. Schuler, F. W. Mohr, and M. Mack, "Transapical Aortic Valve Implantation: Step by Step," The Annals of Thoracic Surgery, vol. 87, pp. 276-283, 2009.
[18] E. Yeniaras, J. Lamaury, N. V. Navkar, D. J. Shah, K. Chin, D. Zhigang, and N. V. T sekos, "Magnetic resonance based control of a robotic manipulator for interventions in the beating heart," presented at Robotics and Automation (ICRA), 2011 IEEE International Conference on, 2011.

[19] A. M. Okamura, C. Richard, and M. R. Cutkosky, "Feeling is believing: Using a force-feedback joystick to teach dynamic systems," Journal of Engineering Education-Washington, vol. 91 , pp. 345-350, 2002.

[20] M. Moreyra and B. Hannaford, "A practical measure of dynamic response of haptic devices," presented at Robotics and Automation, 1998. Proceedings. 1998 IEEE International Conference on, 1998.

[21] K. Kim, J. E. Colgate, J. J. Santos-Munne, A. Makhlin, and M. A. Peshkin, "On the Design of Miniature Haptic Devices for Upper Extremity Prosthetics," Mechatronics, IEEE/ASME Transactions on, vol. 15, pp. 27-39, 2010.

[22] J. S. J. Bolanowski, G. A. Gescheider, R. T. Verrillo, and C. M. Checkosky, "Four channels mediate the mechanical aspects of touch," The Journal of the Acoustical Society of America, vol. 84, pp. 1680-1694, 1988.

[23] M. Li, A. Kapoor, D. Mazilu, and K. A. Horvath, "Pneumatic Actuated Robotic Assistant System for Aortic Valve Replacement Under MRI Guidance," Biomedical Engineering, IEEE Transactions on, vol. 58, pp. 443-451, 2011. 